\title{
Article \\ Greenness Assessment and Synthesis for the Bio-Based Production of the Solvent 2,2,5,5-Tetramethyloxolane (TMO)
}

\author{
Fergal P. Byrne ${ }^{1}$, James H. Clark ${ }^{1}$, Carlo Angelici ${ }^{2}$, Ed de Jong ${ }^{2}\left(\mathbb{D}\right.$ and Thomas J. Farmer ${ }^{1, *(1)}$ \\ 1 Green Chemistry Centre of Excellence, Department of Chemistry, University of York, Heslington, \\ York YO10 5DD, UK; fergal.byrne@york.ac.uk (F.P.B.); james.clark@york.ac.uk (J.H.C.) \\ 2 Avantium, Zekeringstraat 29, 1014 BV Amsterdam, The Netherlands; carlo.angelici@avantium.com (C.A.); \\ ed.dejong@avantium.com (E.d.J.) \\ * Correspondence: thomas.farmer@york.ac.uk; Tel.: +44-(0)-1904-324479
}

Citation: Byrne, F.P.; Clark, J.H.; Angelici, C.; de Jong, E.; Farmer, T.J. Greenness Assessment and Synthesis for the Bio-Based Production of the Solvent 2,2,5,5-Tetramethyloxolane (TMO). Sustain. Chem. 2021, 2, 392-406. https://doi.org/10.3390/ suschem 2030023

Academic Editor: Matthew Jones

Received: 16 June 2021

Accepted: 9 July 2021

Published: 28 July 2021

Publisher's Note: MDPI stays neutral with regard to jurisdictional claims in published maps and institutional affiliations.

Copyright: (c) 2021 by the authors. Licensee MDPI, Basel, Switzerland. This article is an open access article distributed under the terms and conditions of the Creative Commons Attribution (CC BY) license (https:// creativecommons.org/licenses/by/ $4.0 /)$.

\begin{abstract}
TMO) has recently been identified and demonstrated as a safer solvent to replace toluene, $\mathrm{THF}$, and hydrocarbons in a handful of applications. Herein, several bio-based routes to TMO are presented and assessed for greenness, assisted by the CHEM21 Metrics Toolkit and BioLogicTool plots. Using glucose as a common starting point, two chemocatalytic routes and two biochemical routes to TMO were identified and the pathways compared using the aforementioned tools. In addition, bio-based TMO was synthesised via one of these routes; from methyl levulinate supplied by Avantium, a by-product of the sugar dehydration step during the production of 2,5-furandicarboxylic acid. First, methyl levulinate underwent triple methylation using methyl magnesium chloride $(\mathrm{MeMgCl})$ to yield 2,5-dimethylhexane-2,5-diol (DHL) in high yields of $89.7 \%$. Then DHL was converted to high purity TMO ( $>98.5 \%)$ by cyclodehydration using H-BEA zeolites based on the previously reported approach. Bio-based content of this TMO was confirmed by ASTM D6866-20 Method B and found to have $64 \%$ bio-based carbon, well above the threshold of $25 \%$ set by CEN/TC 411 standards and matching the anticipated content. This study represents the first demonstration of a bio-based synthesis of TMO and confirmation of bio-content by accepted standards.
\end{abstract}

Keywords: bio-based solvents; safer solvent; green metrics; platform molecules; solvent substitution

\section{Introduction}

In 2017, the synthesis and testing of 2,2,5,5-tetramethyloxolane (TMO) from 2,5dimethylhexane-2,5-diol (DHL) was published, alongside rationale for its selection as a new non-polar solvent [1]. TMO is a cyclic ether but has properties and performance remarkably similar to toluene in many applications. Importantly, unlike traditional ethers such as diethyl ether, cyclopentylmethylether (CPME) and tetrahydrofuran (THF), TMO does not form peroxides, even when exposed to bubbling air, UV light, and heat [1]. TMO been shown to behave more like toluene than other traditional ethers, 1,4-dioxane, THF and 2-methyltetrahydrofuran (2-MeTHF), in radically-initiated polymerisations [1], Grignard reactions [1], amidation reactions [1], enzyme-catalysed esterification [2] and polycondensation reactions [3]. Solute partitioning experiments showed a stronger affinity for lipophilic protic solutes (fatty acids, fatty alcohols, and phenols) compared to toluene [4,5]. A summary of TMO's properties, as reported in the 2017 article are shown in Table 1.

TMO was initially synthesised from petroleum-derived DHL using H-BEA zeolites, achieving very high yield and selectivity of $>98.5 \%$ in a reactive distillation set-up [1]. The key DHL precursor is currently produced industrially by the reaction of acetylene and acetone, catalysed by potassium isobutoxide ( $\mathrm{KOiBu}$, Scheme 1$)$, in a process patented by BASF [6]. 
Table 1. Properties of TMO compared to toluene and THF.

\begin{tabular}{cccc}
\hline Property & TMO & Toluene & THF \\
\hline Mw $/ \mathrm{g} \mathrm{mol}^{-1}$ & 128.25 & 92.14 & 72.11 \\
Boiling point $/{ }^{\circ} \mathrm{C}$ & $112^{\mathrm{a}}$ & $111^{\mathrm{b}}$ & $66^{\mathrm{b}}$ \\
Melting point $/{ }^{\circ} \mathrm{C}$ & $<-90^{\mathrm{a}}$ & $-95^{\mathrm{b}}$ & $-108^{\mathrm{b}}$ \\
Density $/ \mathrm{g} \mathrm{mL}^{-1}$ & $0.802^{\mathrm{a}}$ & $0.867^{\mathrm{b}}$ & $0.883^{\mathrm{b}}$ \\
Mol. Vol. $/ \mathrm{cm}^{-3} \mathrm{~mol}^{-1}$ & $151.1^{\mathrm{c}}$ & $106.6^{\mathrm{c}}$ & $81.9^{\mathrm{c}}$ \\
Autoignition temperature $/{ }^{\circ} \mathrm{C}$ & $417^{\mathrm{a}}$ & $522^{\mathrm{a}}$ & $321^{\mathrm{a}}$ \\
Lower explosion limit $/ v / v^{\circ}$ & $0.9^{\mathrm{a}}$ & $1.1^{\mathrm{a}}$ & $2.0^{\mathrm{a}}$ \\
$\delta_{\mathrm{D}}^{\mathrm{d}}$ & $15.6^{\mathrm{c}}$ & $18.0^{\mathrm{c}}$ & $16.8^{\mathrm{c}}$ \\
$\delta_{\mathrm{P}}^{\mathrm{e}}$ & $2.3^{\mathrm{c}}$ & $1.4^{\mathrm{c}}$ & $5.7^{\mathrm{c}}$ \\
$\delta_{\mathrm{H}}^{\mathrm{f}}$ & $2.4^{\mathrm{c}}$ & $2.0^{\mathrm{c}}$ & $8.0^{\mathrm{c}}$ \\
$\alpha^{\mathrm{g}}$ & $0.00^{\mathrm{j}}$ & $0.00^{\mathrm{j}}$ & $0.00^{\mathrm{j}}$ \\
$B^{\mathrm{h}}$ & $0.77^{\mathrm{a}}$ & $0.10^{\mathrm{a}}$ & $0.58^{\mathrm{a}}$ \\
$\pi^{* \mathrm{i}}$ & $0.35^{\mathrm{a}}$ & $0.51^{\mathrm{a}}$ & $0.59^{\mathrm{a}}$ \\
$\log P_{(\mathrm{o} / \mathrm{w})}$ & $1.92^{\mathrm{a}}$ & $2.73^{\mathrm{a}}$ & $0.46^{\mathrm{a}}$ \\
\hline
\end{tabular}

a Byrne et al., 2017, previously named 2,2,5,5-tetramethyltetrahydrofuran and abbreviated to TMTHF [1] ${ }^{b}$ PubChem database. ${ }^{c}$ HSPiP (5th Edition 5.1.08). ${ }^{d}$ Hansen solubility parameter for dispersion forces. ${ }^{e}$ Hansen solubility parameter for permanent dipole. ${ }^{\mathrm{f}}$ Hansen solubility parameter for hydrogen-bonding. ${ }^{\mathrm{g}}$ KamletAbboud-Taft parameter for hydrogen-bond donating ability. ${ }^{\text {h }}$ Kamlet-Abboud-Taft parameter for hydrogen-bond accepting ability. ${ }^{i}$ Kamlet-Abboud-Taft parameter for polarity/polarizability. ${ }^{j}$ Assumed value.

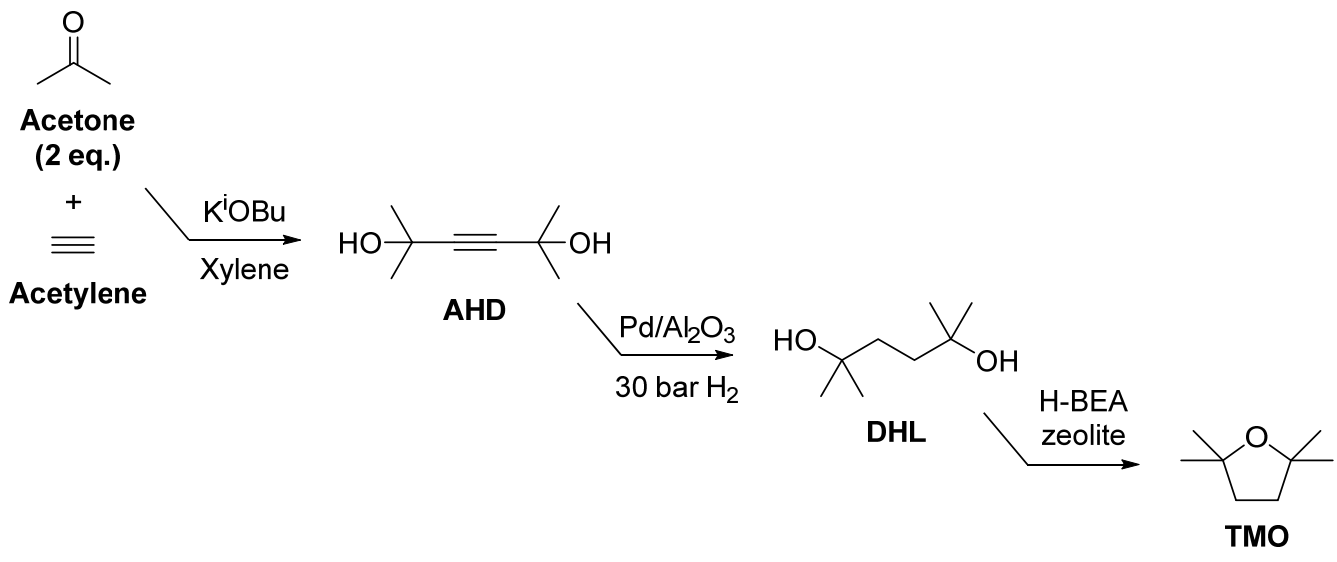

Scheme 1. Synthesis of 2,2,5,5-tetramethyloxolane (TMO) starting from acetone and acetylene.

The bio-based synthesis of TMO was one of the goals of the BBI-JU funded project, ReSolve [7]. Herein, alternative routes to bio-based TMO to the one described above are proposed and compared in terms of greenness with the aid of the CHEM21 Metrics Toolkit [8], and the recently presented BioLogicTool plots [9]. Literature data have been used for many reaction steps, while the authors have carried out certain synthetic steps experimentally in an effort to close any data gaps in the proposed routes (Scheme 2). Finally, bio-based TMO was synthesised using one of the suggested routes, via methyl levulinate, and carbon-14 quantification subsequently confirmed the bio-based content of the DHL and TMO samples (ASTM D6866-20 Method B). Avantium is in the progress of building a global first FDCA plant in Delfzijl, the Netherlands, and therefore industrially relevant quantities of methyl levulinate will become available 2024 onwards. Many potential applications have been identified for levulinic acid and its corresponding methyl ester, but the presented research in this paper is novel to the best of our knowledge [10]. 
<smiles>COCCCCC(C)(O)CCC(C)(C)O</smiles><smiles>CCC(=O)CCC(=O)OC</smiles>

ROUTE 1<smiles>CC(C)(O)CCC(C)(C)O</smiles>

DHL
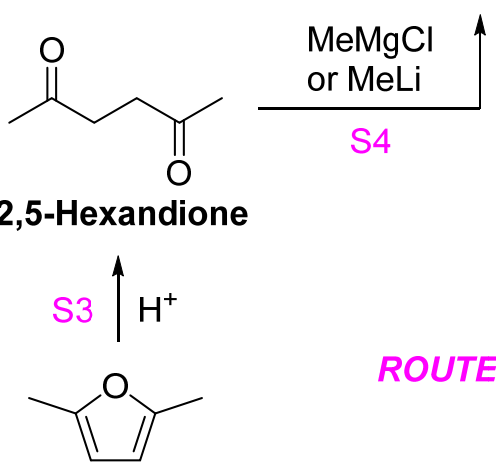

ROUTE 2

\section{2,5-Dimethylfuran}

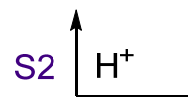

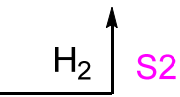

ROUTE 3
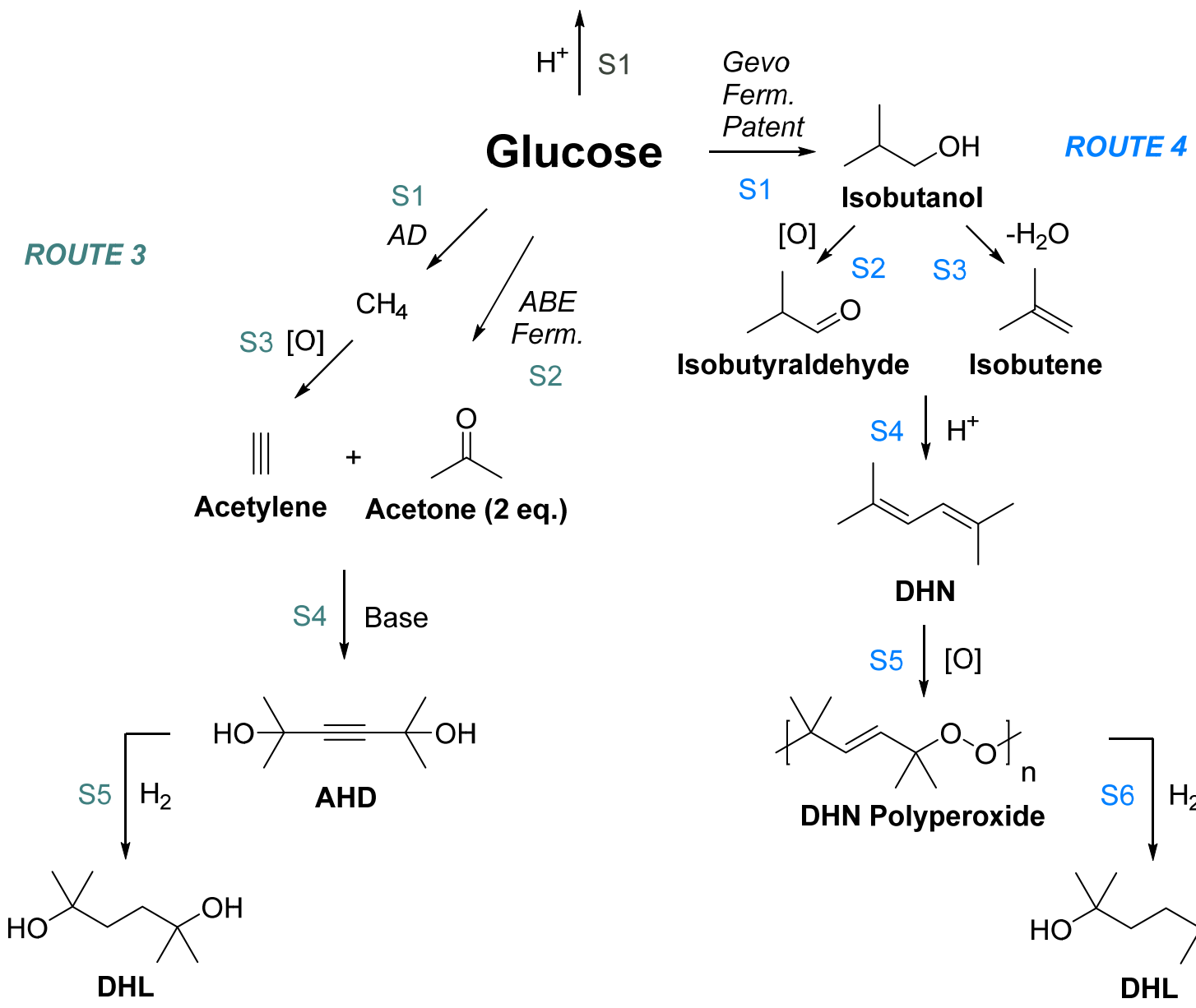


\section{Materials and Methods}

\subsection{Materials}

2,5-dimethylhexa-2,4-diene (DHN), was purchased from Santa Cruz Biotechnology. Methyl levulinate was supplied by Avantium. Methyllithium solution (1.6 M in diethyl ether), $3 \AA$ molecular sieves, methylmagnesium bromide (3 M solution in tetrahydrofuran), ammonium chloride, diethyl ether, 2,5-hexanedione and 10\% Pd/C were purchased from Sigma Aldrich. Sodium chloride and magnesium sulfate and hydrochloric acid were purchased from Fischer Scientific. H-BEA zeolite catalyst (Si/Al ratio 30:1) was supplied by Clariant.

\subsection{Synthesis of 2,5-Dimethylhexane-2,5-Diol from Methyl Levulinate by the Grignard Reaction-Route 1}

Bio-based methyl levulinate $(6.83 \mathrm{~mL}, 50 \mathrm{mmol})$, which had been dried using $3 \AA$ molecular sieves, was added to a $100 \mathrm{~mL}$ round-bottomed flask along with dry tetrahydrofuran $(10 \mathrm{~mL})$ and a magnetic stirring bar. The flask was fitted with a Suba-Seal rubber septum and the mixture was degassed. A balloon was fitted to the rubber septum and the temperature of the mixture was reduced to $0{ }^{\circ} \mathrm{C}$, at which point methylmagnesium bromide ( $3 \mathrm{M}$ solution in tetrahydrofuran, $53.3 \mathrm{~mL}, 3.2$ equivalents) was added dropwise using a syringe. The reaction mixture was stirred for $2 \mathrm{~h}$ at room temperature, after which the reaction was quenched using ammonium chloride solution. The mixture was transferred to a $250 \mathrm{~mL}$ separating funnel along with $25 \mathrm{~mL}$ diethyl ether. The organic layer was collected and washed twice with brine $(10 \mathrm{~mL})$. The aqueous layer was then back-extracted twice using diethyl ether $(10 \mathrm{~mL})$. The organic fractions were combined, dried with magnesium sulfate, and the solvent was removed in vacuo to yield crude 2,5dimethyl-2,5-hexandiol (DHL) as an off-white powder. The powder could be recrystallised in diethyl ether to produce clear needles of 2,5-dimethyl-2,5-hexandiol with a yield of $89 \% .{ }^{1} \mathrm{H}$ NMR (400 MHz, $\left.\mathrm{CDCl}_{3}\right): \delta=1.18$ (s, $\left.12 \mathrm{H}, 4 \times\left(-\mathrm{CH}_{3}\right)\right), 1.53\left(\mathrm{~s}, 4 \mathrm{H}, 2 \times\left(-\mathrm{CH}_{2}-\right)\right) ;{ }^{13} \mathrm{C}$ $\operatorname{NMR}\left(400 \mathrm{MHz}, \mathrm{CDCl}_{3}\right): \delta=29.35,39.17,71.37$; MS (ESI): m/z 147.14 [1 $\left.1^{+}\right], 169.12\left[\mathrm{Na}^{+}\right]$; Bio-based carbon content: $64 \%$.

\subsection{Synthesis of 2,5-Dimethylhexane-2,5-Diol from 2,5-Hexanedione Using Methyllithium-Route 2}

To a flame-dried, argon-filled round-bottomed flask was added 2,5-hexanedione $(1 \mathrm{~mL}, 9 \mathrm{mmol})$ and $20 \mathrm{~mL}$ dry and degassed diethyl ether. The mixture was further purged with argon using the freeze-thaw method. The temperature was reduced to $-78^{\circ} \mathrm{C}$ and methyllithium solution (1.6 M in diethyl ether, $10 \mathrm{~mL}, 16 \mathrm{mmol}$ methyllithium) was added dropwise. The reaction mixture was allowed to stir for $2 \mathrm{~h}$ at $-78^{\circ} \mathrm{C}$ before being brought to room temperature and stirred overnight. The reaction was then quenched by slowly adding water at $0{ }^{\circ} \mathrm{C}$, before $10 \mathrm{~mL}$ of $2 \mathrm{M}$ hydrochloric acid was added dropwise. The mixture was transferred to a separating funnel where the organic layer was collected and washed with brine $(2 \times 10 \mathrm{~mL})$ and the combined aqueous fractions were back-extracted using diethyl ether $(2 \times 10 \mathrm{~mL})$. The combined organic fractions were dried using magnesium sulphate and concentrated in vacuo to yield crude 2,5-dimethyl-2,5-hexandiol as an offwhite powder. The powder could be recrystallised in diethyl ether to produce clear needles of 2,5-dimethyl-2,5-hexandiol with a yield of $71 \% .{ }^{1} \mathrm{H}$ NMR $\left(400 \mathrm{MHz}, \mathrm{CDCl}_{3}\right): \delta=1.24$ (s, $\left.12 \mathrm{H}, 4 \times\left(-\mathrm{CH}_{3}\right)\right), 1.58\left(\mathrm{~s}, 4 \mathrm{H}, 2 \times\left(-\mathrm{CH}_{2}-\right)\right) ;{ }^{13} \mathrm{C} \mathrm{NMR}\left(400 \mathrm{MHz}, \mathrm{CDCl}_{3}\right): \delta=29.54,37.99,70.81$.

\subsection{Oxidation of 2,5-Dimethylhexa-2,4-Diene (DHN)-Route 4}

DHN (5 g, $45.4 \mathrm{mmol})$, assumed petrochemical derived, was added to a $25 \mathrm{~mL}$ roundbottomed flask along with 2,2,5,5-tetramethyloxolane (TMO, $10 \mathrm{~mL}$ ) and a magnetic stirring bead. The flask was sealed with a rubber septum which was pierced with a long syringe needle connected to a compressed air source (air inlet). This syringe needle was submerged in the reaction mixture. A second and third syringe needle were then inserted into the rubber septum (air outlet) but kept above the reaction mixture level. The mixture was

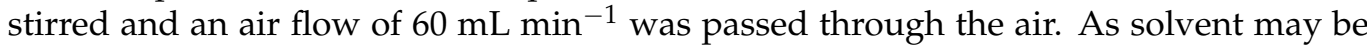


lost by evaporation out the air outlet during the reaction, fresh solvent can be added as required to maintain a viscosity that can be stirred by the magnetic stirring bead. Reaction progress was monitored by ${ }^{1} \mathrm{H}$ NMR spectroscopy. Upon completion of the reaction, the solvent was removed in vacuo to yield DHN polyperoxide with a yield of $97.5 \% .{ }^{1} \mathrm{H}$ NMR $\left(400 \mathrm{MHz}, \mathrm{CDCl}_{3}\right): \delta 5.66(\mathrm{~s}, 2 \mathrm{H}, 2 \times(-\mathrm{CH}=)), 1.26\left(\mathrm{~s}, 12 \mathrm{H}, 4 \times\left(-\mathrm{CH}_{3}\right)\right)$.

\subsection{Hydrogenation of DHN Polyperoxide-Route 4}

DHN polyperoxide $(1 \mathrm{~g}), 2,2,5,5$-tetramethyloxolane $(10 \mathrm{~mL})$ and $10 \% \mathrm{Pd} / \mathrm{C}(500 \mathrm{mg})$ were added to a $20 \mathrm{~mL}$ Hastelloy steel autoclave. The autoclave was purged with hydrogen and then pressurised with hydrogen (10 bar). The reaction mixture was stirred for $18 \mathrm{~h}$ at $18{ }^{\circ} \mathrm{C}$ before being depressurised, filtered, and analysed by NMR spectroscopy.

\subsection{Synthesis of 2,2,5,5-Tetramethyloxolane (TMO) from 2,5-Dimethylhexane-2,5-Diol (DHL)}

2,5-dimethylhexane-2,5-diol (DHL, $10 \mathrm{~g}, 68.4 \mathrm{mmol}$ ) was added to a $25 \mathrm{~mL}$ roundbottomed flask fitted with Vigreaux distillation apparatus along with H-BEA zeolite catalyst ( $\mathrm{Si} / \mathrm{Al}$ ratio 30:1) and a magnetic stirring bead. The reaction mixture was heated to $140{ }^{\circ} \mathrm{C}$, at which point the 2,5-dimethylhexane-2,5-diol melted and began to react. Upon the formation of 2,2,5,5-tetramethyloxolane and water, the mixture began to reflux. The distillate was collected as two phases. The organic phase was further purified by distillation to yield 2,2,5,5-tetramethyloxolane with a purity of $99 \%$ as confirmed by GC-FID. ${ }^{1} \mathrm{H}$ NMR $\left(400 \mathrm{MHz}, \mathrm{CDCl}_{3}\right): \delta 1.81\left(\mathrm{~s}, 4 \mathrm{H}, 2 \mathrm{x}\left(-\mathrm{CH}_{2}-\right)\right), 1.21\left(\mathrm{~s}, 12 \mathrm{H}, 4 \mathrm{x}\left(-\mathrm{CH}_{3}\right)\right) ;{ }^{13} \mathrm{C} \mathrm{NMR}(400 \mathrm{MHz}$, $\mathrm{CDCl}_{3}$ ): $\delta$ 29.75, 38.75, 80.75; MS (ESI): $\mathrm{m} / \mathrm{z} 129.13$ [1+ ${ }^{+}$; Bp: $112{ }^{\circ} \mathrm{C}$; bio-based carbon content: $64 \%$.

\subsection{Bio-Based Carbon Content Testing}

2,5-dimethylhexane-2,5-diol (DHL) and 2,2,5,5-tetramethyloxolane (TMO) from Route 1 above were tested according to ASTM D6866-20 Method B at the laboratory of BETA Analytics in Florida.

\section{Results}

Several synthetic routes to DHL, the precursor to TMO, from biomass were identified and can be seen in Scheme 2. A high yielding, essentially quantitative, synthesis of TMO from DHL has previously been reported [1], so no further comments are made about that final step of the synthesis in this work. Glucose is illustrated as the starting point for simplicity, but fructose, starch, cellulose or lignocellulosic biomass could also have been shown [11,12]. Indeed, lignocellulosic biomass would be the ideal source of glucose as it is a second-generation biomass source, although an additional pre-treatment step to what is shown in Scheme 2 would be required [13]. Two routes (Routes 1 and 2) proceed chemocatalytically via 5-(hydroxymethyl)furfural (HMF) and two routes (Routes 3 and 4) proceed predominately biochemically. The biochemical conversion of glucose to platform molecules methane, acetone and isobutanol in Routes 3 and 4 will not be assessed for greenness in this report due to insufficient data being available, but they will be included in the step count in Table 2. Table 2 shows the yield, conversion, selectivity, atom economy (AE), reaction mass efficiency (RME) and process mass intensity (PMI). Below is a detailed description of each Route 1-4 followed by a discussion of their accompanying BioLogicTool [9] and metric assessments. Metric assessment was performed using the CHEM21 Metric Toolkit [8]. The BioLogicTool is a new graphical tool that plots pathways from feedstock through to products against axis of $\%$ heteroatom content by mass versus molar mass [9]. The BioLogicTool allows for comparisons of potential routes prior to synthesis (Figure 1) and specifically looks at how rational each route is in terms of optimum use of the abundant heteroatoms in the biomass feedstock. 
Table 2. Reaction metrics of each step (SX) of each Route (1 to 4) to DHL and subsequently to TMO as set out in Scheme 2.

\begin{tabular}{|c|c|c|c|c|c|c|}
\hline & Yield/\% & Conversion $/ \%$ & Selectivity/\% & $\mathrm{AE} * / \%$ & RME */\% & PMI * \\
\hline \multicolumn{7}{|c|}{ Route 1} \\
\hline S1 & $\mathrm{n} / \mathrm{a}$ & $\mathrm{n} / \mathrm{a}$ & $\mathrm{n} / \mathrm{a}$ & $\mathrm{n} / \mathrm{a}$ & $\mathrm{n} / \mathrm{a}$ & $\mathrm{n} / \mathrm{a}$ \\
\hline S2 & $\mathrm{n} / \mathrm{a}$ & $\mathrm{n} / \mathrm{a}$ & $\mathrm{n} / \mathrm{a}$ & $\mathrm{n} / \mathrm{a}$ & $\mathrm{n} / \mathrm{a}$ & $\mathrm{n} / \mathrm{a}$ \\
\hline S3 & $\mathrm{n} / \mathrm{a}$ & $\mathrm{n} / \mathrm{a}$ & $\mathrm{n} / \mathrm{a}$ & 97 & 1 & 78 \\
\hline S4 & 90 & 100 & 90 & 41 & 37 & 23 \\
\hline \multicolumn{7}{|c|}{ Route 2} \\
\hline S1 & 56 & 88 & 64 & 70 & 39 & 36 \\
\hline S2 & 71 & 100 & 71 & 75 & 53 & 69 \\
\hline S3 & 99 & 100 & 99 & 100 & 99 & 12 \\
\hline S4 & 71 & 100 & 71 & 92 & 63 & 110 \\
\hline \multicolumn{7}{|c|}{ Route 3} \\
\hline S1 & $\mathrm{n} / \mathrm{a}$ & $\mathrm{n} / \mathrm{a}$ & $\mathrm{n} / \mathrm{a}$ & $\mathrm{n} / \mathrm{a}$ & $\mathrm{n} / \mathrm{a}$ & $\mathrm{n} / \mathrm{a}$ \\
\hline S2 & $\mathrm{n} / \mathrm{a}$ & $\mathrm{n} / \mathrm{a}$ & $\mathrm{n} / \mathrm{a}$ & $\mathrm{n} / \mathrm{a}$ & $\mathrm{n} / \mathrm{a}$ & $\mathrm{n} / \mathrm{a}$ \\
\hline S3 & 63 & 70 & 90 & 81 & 51 & 2 \\
\hline S4 & 92 & 98 & 94 & 100 & 94 & 8 \\
\hline S5 & 96 & 100 & 99 & 100 & 99 & 3 \\
\hline \multicolumn{7}{|c|}{ Route 4} \\
\hline S1 & $\mathrm{n} / \mathrm{a}$ & $\mathrm{n} / \mathrm{a}$ & $\mathrm{n} / \mathrm{a}$ & $\mathrm{n} / \mathrm{a}$ & $\mathrm{n} / \mathrm{a}$ & $\mathrm{n} / \mathrm{a}$ \\
\hline S2 & 50 & 50 & 100 & 97 & 49 & 2 \\
\hline S3 & 96 & 99 & 97 & 76 & 73 & 1 \\
\hline $\mathrm{S} 4{ }^{\circledR}$ & $95(33)^{\circledR}$ & $100(35)^{@}$ & $95(95)^{@}$ & $86(86)^{@}$ & $82(26)^{@}$ & $1(3.5)^{@}$ \\
\hline S5 & 98 & 98 & 100 & 100 & 98 & 2 \\
\hline S6 & 87 & 100 & 87 & 100 & 84 & 8 \\
\hline \multicolumn{7}{|c|}{$\begin{array}{c}\text { DHL to } \\
\text { TMO }\end{array}$} \\
\hline & 99 & 100 & 99 & 88 & 87 & 1 \\
\hline
\end{tabular}

${ }^{*}$ Metrics calculated using the CHEM21 Metrics Toolkit, the spreadsheet used for these calculations is available as a separate supplementary information file titled "CHEM21 Metrics-All Routes". AE = atom economy; RME = reaction mass efficiency; PMI = process mass intensity. ${ }^{\circledR}$ The numbers outside the brackets are for a hypothetical multi-pass through the quoted flow reactor of Route 4 S4, the number inside the brackets are for a single pass through the flow reactor.

\subsection{Route 1-Via Levulinic Acid or Methyl Levulinate}

Route 1 can start both from levulinic acid as well as from methyl levulinate (ML). Levulinic acid is a desired platform molecule on its own $[10,14]$, while ML is produced by Avantium as a by-product from their proprietary $Y X Y^{\circledR}$ process to produce 2,5-furandicarboxylic acid (FDCA) from fructose [15]. Triple methylation of the carbonyl groups of levulinic acid yields DHL. In addition, levulinic acid can be esterified prior to methylation, which helps to maintain dry conditions during the reaction. Route 1 requires few steps, but the methylation of carbonyl groups raises an issue from a green perspective, as outlined below.

Levulinic acid is produced easily from biomass and there are many reports of its synthesis in the literature, either from saccharides and polysaccharides, HMF or as a by-product in the production of other chemicals [14]. However, sufficient data for the metrics calculation of its synthesis from glucose are unavailable in the literature, as only conversions based on HPLC analysis are presented [14]. In the current work, ML was supplied by Avantium, this produced as a by-product in the sugar dehydration step in their YXY process for FDCA production. Again, this makes traditional green metrics more complicated to apply, but benefits can clearly be seen [16]. Most obvious, an underutilized potential side-stream is valorised, reducing the waste of the YXY process. Furthermore, the inputs (catalysts, solvents, etc.) can be divided by the relative masses of the products for the calculation of RME and PMI for each product. 
Route 1

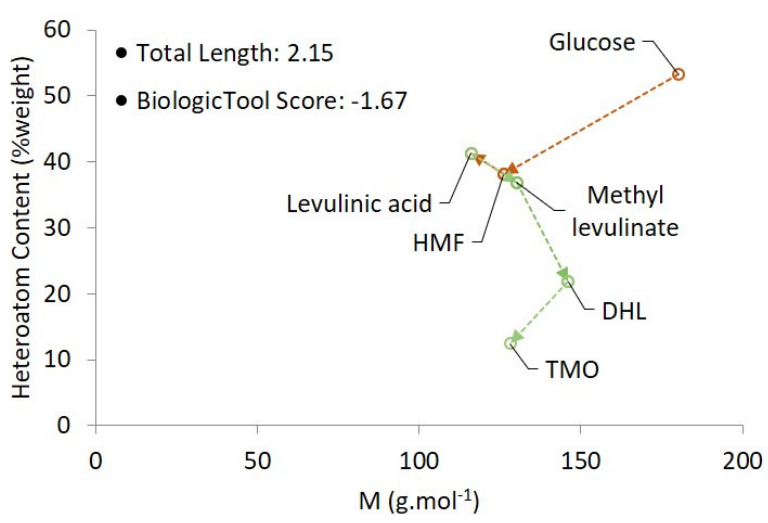

Route 3

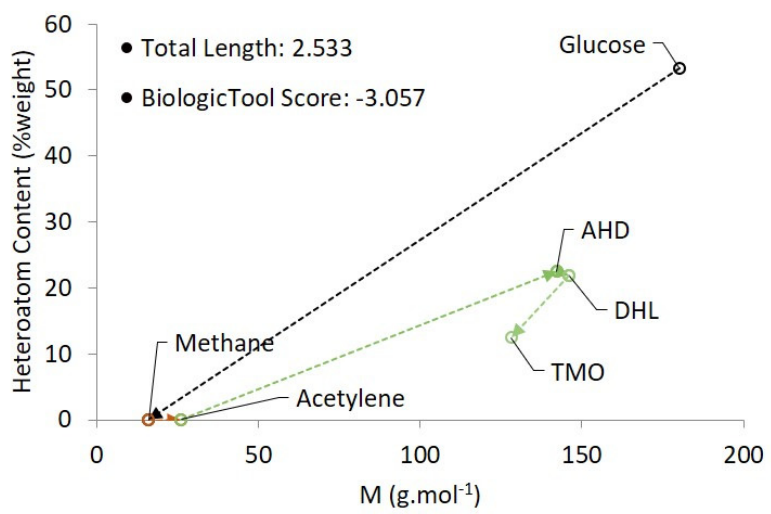

Route 2

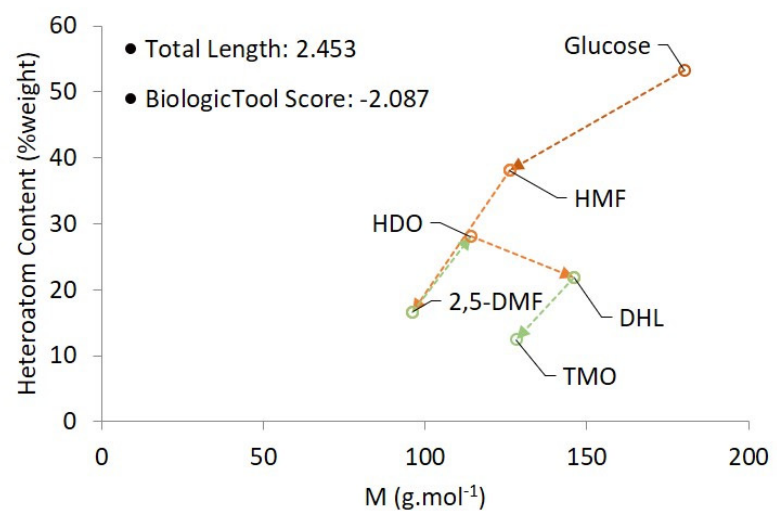

Route 4

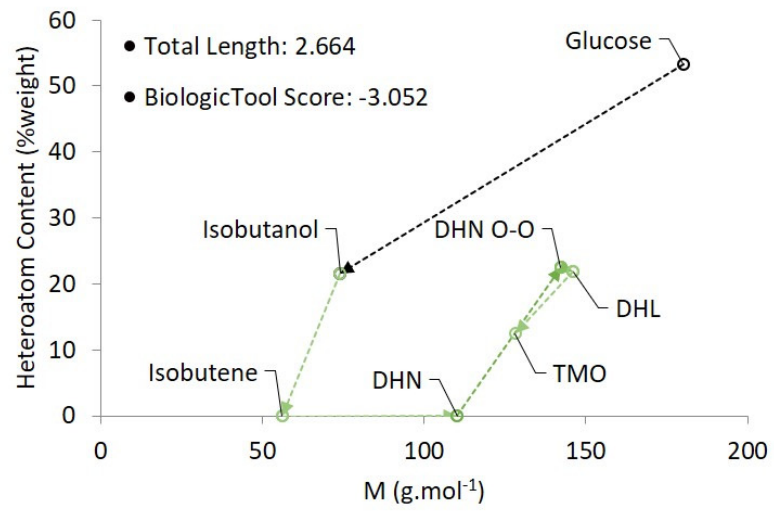

Figure 1. Plots generated by the BioLogicTool, showing the pathways of each route 1-4 to TMO from a common glucose feedstock for each of the four routes. The colour of the arrow is determined by the yield of that step (see Table 2); green being the highest, red being the lowest, and black where no or insufficient data was available. The BioLogicTool does not consider the possibility that a synthetic pathway requires the merging of two routes at any point in the synthesis. As such, the larger numerical value (ignoring minus sign) for the BioLogicTool score and Total Length for any convergent pathway has been presented, this deemed more representative where a convergent synthesis is used. BioLogicTool plots were prepared using the spreadsheet available as supplementary information accompanying the original 2019 article by Lie et al. [9].

In aqueous conditions, levulinic acid is formed instead of ML. Levulinic acid can react with alcohols to produce levulinate esters such as ML [17]. However, compared with levulinic acid, ML is easier to isolate and purify due to its significantly lower boiling point. ML is therefore the preferred starting material because it is easier to maintain dry conditions compared to levulinic acid in the subsequent moisture sensitive steps. Metrics for the esterification process are given in Table 2 based on the work of Pan et al. [18].

There are many methods of methylation of carbonyl groups, with the Grignard reaction being among the most commonly used at lab-scale. Complete conversion of the starting material was observed by the Grignard reaction and a DHL yield of $90 \%$ was achieved (Scheme 3). Subsequent ring closure of DHL to produce TMO was performed without further purification. A selectivity to TMO of $>98.5 \%$ was obtained in a continuous reactive distillation, catalysed by H-BEA zeolites as previously described [1], this resulted in the isolation of the partially bio-based TMO of high purity as confirmed by NMR spectroscopy (ESI, Figure S1)

Even if the methide anion was petroleum-derived, the bio-based content of DHL produced by this route would be $62.5 \%$ (five out of eight carbons in the final structure are bio-based as can be seen in Scheme 3), well above the $25 \%$ bio-based carbon required by the CEN/TC 411 (Bio-based products). Bio-based carbon content was measured by ASTM 
D6866-20 Method B and showed 64\% bio-based carbon content in both the DHL and TMO subsequently produced, consistent with theoretical calculations.

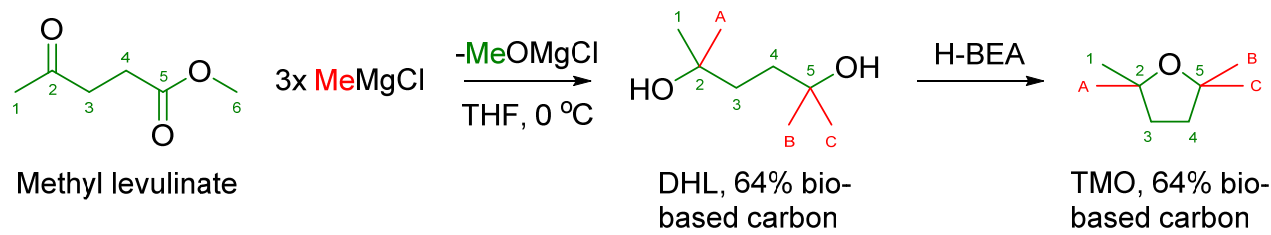

Scheme 3. Synthesis of bio-based TMO via DHL from methyl levulinate. Bio-based carbon is shown in green, petroleum-derived carbon is shown in red.

While the Grignard reaction allows the production of bio-based DHL in high yields and high purity (by NMR spectroscopy, ESI Figures S2 and S3), the AE and RME are quite low at $41 \%$ and $37 \%$ respectively (ESI, CHEM21 Metrics spreadsheet, tab R1 S4). In particular, large amounts of hazardous solvent were required and stoichiometric amounts of magnesium salts produced which are difficult to recover and reuse. Furthermore, dry conditions were required, adding to the complexity of this reaction, but partially overcome through the use of ML that it is easier to dry than levulinic acid. Ultimately, Route 1 will only become suitable for efficient large-scale production of bio-based TMO when more efficient and preferably bio-based methylation routes are developed.

\subsection{Route 2-Via 2,5-Dimethylfuran}

Route 2 is a chemocatalytic route in which glucose is first dehydrated to HMF, which in turn is hydrogenated to 2,5-dimethylfuran. 2,5-Dimethylfuran can be easily converted via hydrolysis to 2,5-hexanedione (HDO) by treatment with acid and water. Methylation of HDO forms DHL which can be ring-closed to generate TMO.

The first step of this route required the production of HMF from a hexose carbohydrate source, such as glucose, cellulose or starch. The production of HMF is not trivial due to its instability in air and light, but AVA BioChem has begun production on a 20 tonne per annum scale [19]. The reaction is carried out at $220^{\circ} \mathrm{C}$ and $22 \mathrm{bar}$ in acidic conditions, and an in situ biomass power plant is proposed to provide energy for the chemistry [19]. The exact metrics of AVA's process cannot be calculated due to lack of data, but literature values are used to give approximate metrics in supplementary CHEM21 spreadsheet, tab R2 S1 [20]. In any case, the large-scale production of HMF represents a major breakthrough in bio-based commodity production and should be viewed positively overall due to the opportunities it will open [12].

2,5-Dimethylfuran can be easily converted to HDO in excellent isolated yields in acidic aqueous conditions. Yang et al. reported $99 \%$ yields using a biphasic system of methyl isobutyl ketone (MIBK) and acidic water [21]. Sulfuric acid was found to be the best catalyst and MIBK could extract HDO from the acidic aqueous phase prior to self-aldol reactions occurring [21]. This step is highly efficient, achieving an atom economy of $100 \%$ and RME of 99\% (supplementary CHEM21 spreadsheet, tab R2 S3), meaning no purification is required prior to the next step (Table 2).

HDO can be converted to DHL in a similar manner to methyl levulinate-by methylation at the carbonyl groups (Scheme 4). HDO has the benefit of requiring only two equivalents of methylating agent, which can be sourced from methylating agents such as Grignard reagents or methyllithium. This results in an AE of $92.4 \%$ and RME of $62.5 \%$ (supplementary CHEM21 spreadsheet, tab R2 S4). The AE is significantly better than when a Grignard reagent is used, but more controlled conditions complicate the process significantly compared to the Grignard reaction. 


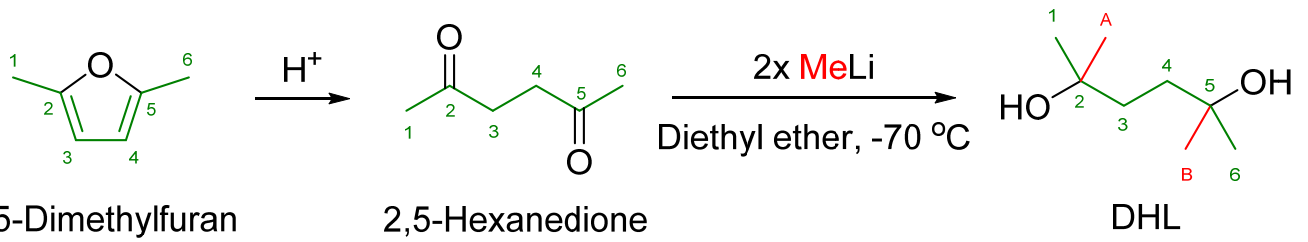

Scheme 4. Synthesis of 2,5-dimethyl-2,5-hexanediol (DHL) via 2,5-dimethylfuran and 2,5-hexanedione.

When performing this route in the lab we selected methyllithium as the methide ion source to compare the process to that of the aforementioned Grignard reaction chosen for Route 1 . HDO was used in a slight excess as we observed that methyllithium would act like a base and deprotonate HDO, causing aldol-type reactions to occur. It was found that the conditions required for methyllithium were much more sensitive than for the Grignard reaction. Dry glassware was required, reagents needed to be carefully dried and degassed, and low reaction temperatures of $-78{ }^{\circ} \mathrm{C}$ were required to obtain high selectivity $(\sim 90 \%)$. If the stringency of the conditions was eased even slightly, by not flame drying glassware or increasing temperature $\left(-20^{\circ} \mathrm{C}\right)$, then selectivity dramatically suffered as shown by NMR spectroscopy (ESI, Figures S4 and S5). In addition, like the Grignard reaction, a stoichiometric amount of lithium containing waste is produced. As such, this route is unlikely to be suitable for commercial production of TMO. Although HDO is readily bioderivable, large amounts of hydrogen gas are required to form the 2,5-dimethylfuran from HMF, and the source of HDO from chemical suppliers is unknown so the DHL and TMO produced by this route was not tested for bio-based content.

\subsection{Route 3-Via Bio-Acetylene and Bio-Acetone}

Route 3 is a bio-based equivalent to the established petroleum derived synthesis of DHL (Scheme 2). Acetone (likely sourced from the cumene process) [22,23] and methane (likely sourced from natural gas) [24] are the current starting materials for this process. Conveniently, both of these compounds are two of the most established bio-based platform chemicals-acetone is one product of the $\mathrm{ABE}$ (acetone-butanol-ethanol) fermentation process $[25,26]$ (this was the primary source of acetone prior to the first world war) while methane is a product of anaerobic digestion (AD) $[27,28]$. A slight increase in cost between petroleum-derived and bio-based versions of acetone and methane can be expected initially, but increasing legislation, economies of scale, carbon tax and improving technology will likely make the bio-based production of acetone and methane competitive over time.

In this hypothetical bio-based route, methane is first produced by AD and then partially oxidised, generating acetylene (and syngas) [29,30]. Acetylene is reacted with two equivalents of $\mathrm{ABE}$ fermentation-sourced acetone using xylene as a solvent and potassium isobutoxide $(\mathrm{KO} i \mathrm{Bu})$ as a recoverable base to yield an alkyne hexanediol (AHD, Scheme 5). AHD is hydrogenated to produce $100 \%$ bio-based DHL. The benefit of this route is that the processes are all established, and a simple switch to bio-based methane and acetylene is all that is required.

The first reactions in this route involve the biochemical production of methane and acetone from glucose. Biochemical processes will not be assessed in this work as other publications with assessments of biochemical production of chemicals are available in the literature [31-33]. The oxidation of methane is a well-established process for the production of acetylene (Route 3 S1) [34]. Although acetylene yields are modest ( 25\%), work continues on the improvement of oxidative coupling of methane today using new reactor technology. For example, Khoe Dinh et al. reported methane conversions of over $70 \%$ with over $90 \%$ selectivity for acetylene using a novel rotating arc driven by AC electrical power [29].

The production of DHL from acetylene and acetone was patented by BASF in 2005 (Route 3 S2) [6]. This involved the coupling of acetylene and two equivalents of acetone to produce an alkyne, AHD (2,5-dimethylhex-3-yne-2,5-diol), followed by hydrogenation of AHD to yield DHL [6]. The yield and RME of the coupling step are excellent at $92 \%$ and 
$93 \%$, respectively, and $\mathrm{AE}$ is $100 \%$ (Table 2). $\mathrm{KO}^{i} \mathrm{Bu}$ in a suspension of xylene is used as the base in the reaction. Although stoichiometric amounts of base are required to deprotonate the acetylene, a simple process for the recovery and reuse of $\mathrm{KO}^{i} \mathrm{Bu}$ is also covered in the patent [6]. This means that the potential production of huge amounts of waste is eliminated. It appears that xylene is selected as a solvent due to its polarity and relative inertness under reaction conditions. If this is the case, there are many greener solvents with similar solubility properties that could be used instead, the most obvious being TMO itself. The hydrogenation of AHD was reported as being done in flow in solventless conditions and used $\mathrm{Pd} / \mathrm{Al}_{2} \mathrm{O}_{3}$ as the catalyst [6]. A yield of $99 \%$ was achieved with $100 \%$ atom economy (Table 2). Finally, the ring closure of DHL to produce TMO is completed as previously reported.

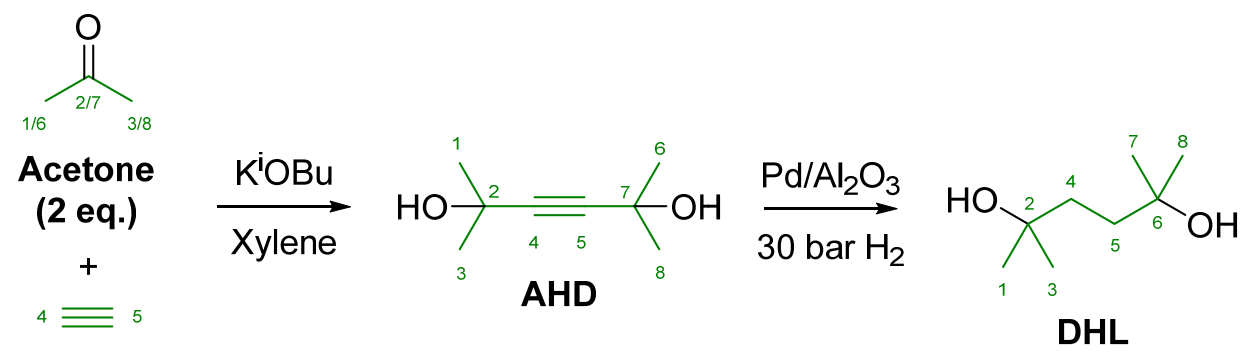

Acetylene

Scheme 5. Synthesis of DHL from acetone and acetylene via AHD.

Route 3 to TMO involves six steps, with the first two being the biochemical conversion of glucose to methane and acetone in well-established processes. Once acetone and acetylene are produced, the coupling reaction, hydrogenation reaction, and final ring closure to produce TMO can all be carried out in series, without the need for purification in between. All steps are either catalytic or use recoverable and reusable reagents $\left(\mathrm{KO}^{i} \mathrm{Bu}\right)$ which significantly increases the likelihood of commercial viability of the overall process. However, an inability to source bio-based acetone and methane meant we were unable to perform this reaction and isolate bio-based TMO from this route.

\subsection{Route 4-Via Isobutanol}

Route 4 also relies on a key biochemical step, but one which now uses the platform molecule isobutanol produced via fermentation (Scheme 2). Gevo and Butamax have been developing the biosynthetic pathway to isobutanol for the past 10 years for use as a transport fuel. Furthermore, isobutanol can be used as a platform molecule to produce a wide range of other chemicals such as isobutene [35], isobutyraldehyde [36], isobutyric acid [37], isooctane [38] and para-xylene [39].

Route 4 requires both the partial oxidation of isobutanol to isobutyraldehyde and the dehydration of isobutanol to isobutene (Scheme 2) [40]. Isobutyraldehyde and isobutene can then be coupled to produce a fully bio-based diene, 2,5-dimethylhexa-2,4-diene (DHN) [40]. DHN is then oxidised to produce peroxide oligomers that can be hydrogenated to yield DHL precursor of TMO (this work).

Underpinning the proposed Route 4 is a Gevo patent from 2014 reporting the synthesis of DHN in a series of high yielding flow processes [40]. In the first set of flow reactions, isobutanol is partially oxidised to isobutyraldehyde using a supported $\mathrm{CuO}$ catalytic system and leaving some residual unreacted isobutanol (Route 4 S1). Only partial conversion is required and complete selectivity for the desired product is achieved, with a high AE of 97.3\% (Table 2). The small loss of $\mathrm{AE}$ is due to the elimination of hydrogen during the oxidation process. However, it will be seen that this hydrogen can in theory be recovered and reused in a later step.

The isobutanol/isobutyraldehyde mixture is then passed over a solid dehydration catalyst generating a highly pure mixture of isobutene and isobutyraldehyde (Route 4 S2). 
Again, almost complete conversion and selectivity for isobutene is achieved, with the AE only being affected by the loss of water.

Isobutene and isobutyraldehyde are coupled using niobic acid to produce DHN [Route 4 S3]. Reported conversion is low at 35\%, but as selectivity is high (95\%) (Table 2 and CHEM21 spreadsheet, tab "R4 S3-one pass"). If unreacted starting material is simply recycled back via a flow system then a high atom economy and RME of $82 \%$ (Table 2 and CHEM21 spreadsheet, tab "R4 S3-multi-pass"). However, it does not appear that Gevo are currently pursuing this synthesis as DHN is not listed in their catalogue of commercial products at the time of writing.

$\mathrm{DHN}$ is an interesting molecule in that it can oxidise in air at room temperature to form oligomers of a stable polyperoxide (DHN polyperoxide, Scheme 6) [S4]. No solvent was used in the oxidation step as reported by Griesbaum et al. [41], but an extraction using methanol was required to remove unreacted diene. As TMO does not easily oxidise (unlike other common ethers such as THF and diethyl ether) [1], we successfully used TMO itself as the polymerisation solvent in the current work, usually commercially available and assumed petrochemical-derived DHN (NMR spectra in ESI, Figure S6. Our approach using TMO as solvent maintained a low viscosity of the mixture and eliminated the need of a methanol extraction. The process involved the slow bubbling of air through the mixture slowly overnight, obtaining almost complete conversion of DHN (97.5\%) and minimal TMO losses. The atom economy of this step is $100 \%$, and the polyperoxide was isolated in high purity as shown by NMR spectroscopy (ESI, Figure S7)

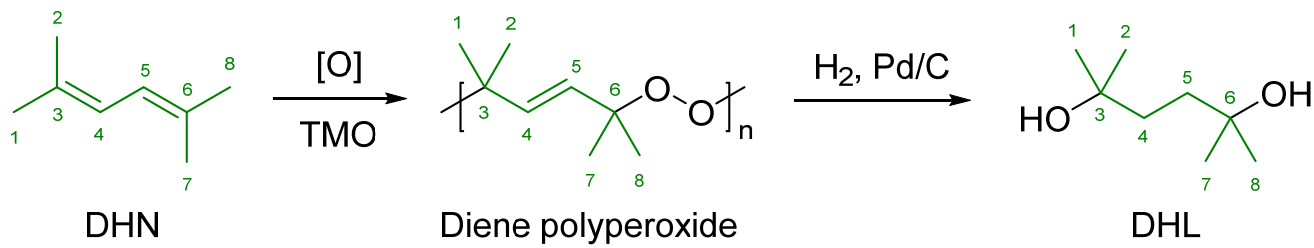

Scheme 6. Oxidation of 2,5-dimethylhexane-2,4-diene (DHN) and subsequent hydrogenation to produce DHL.

Hydrogenation of the DHN polyperoxide yields DHL at almost complete conversion, as described by Griesbaum et al. [41]. In that work, THF was used as the solvent for the hydrogenation step with yields of $90 \%$. However, TMO could be a suitable solvent for the hydrogenation in a neat system where the final product is used as the solvent in several reaction steps. However, when this mixture was transferred to an autoclave along with $\mathrm{Pd} / \mathrm{C}(10 \%)$ catalyst and pressurised with hydrogen gas (10 bar) in similar conditions to those reported by Griesbaum et al. [41], no reaction was observed. The reasons for this are unclear as there is no obvious barrier to the reaction occurring, but further research is clearly required to develop this pathway to DHL. It is also possible that hydrogen produced from the first step of this route (oxidation of isobutanol) can be recovered and used directly in this hydrogenation step. Finally, the DHL/TMO mixture can be exposed to H-BEA catalysts without the need for DHL isolation and still produce more TMO, completing a neat system where TMO is the solvent for its own production in several steps.

Overall, despite requiring a greater number of steps than the other routes, all steps are catalytic and many of the steps are carried out in flow without the need for any purification in between. When combined with the general benignity of each step, this route has many green aspects, as can be seen in Table 2.

\section{Discussion}

The greenness of any synthetic route to any product should be viewed holistically, i.e., no one metric is enough to rate the greenness of a process. In this section we assess the greenness using all relevant tools available. Table 2 shows the CHEM21 metrics for each step of the four routes to TMO, while the BioLogicTool shows that the BioLogic score and 
Total BioLogic Length in Figure 1. Further questions should be asked however, which are not measurable by a score:

- $\quad$ Are toxic reagents used?

- Is the synthesis moisture- or air-sensitive?

- Are critical elements required?

- How quickly does the reaction proceed?

- Can it be carried out in flow?

Some of these questions are answered in the CHEM21 Metrics Toolkit spreadsheet, and can be seen viewed as a supplementary file with a separate tab for each step of each route. The total yield of final product as a percentage of biomass would be another highly informative metric for the mass efficiency of the processes, but unfortunately values for the yield is not available for each step of all routes, so a comparative overall route yield could not be calculated.

From the four routes to TMO proposed in this work, two proceeded chemocatalytically and two involved biochemical transformations. The two chemocatalytic routes require fewer steps from glucose, which is usually a good indicator of greenness as each additional unit operation likely carries greater energy and material demands. The BioLogicTool shows that the BioLogic score (the tortuosity of a route) and Total BioLogic Length (summation of the path lengths) of the chemocatalytic routes are better (i.e., lower values, noting that the minus symbol describes the vector direction, not length) than the biochemical routes. These conclusions from the BioLogicTool are roughly consistent with the fewer steps required from the shared glucose feedstock (Figure 1). Despite the better appearance of the chemocatalytic routes at this point, the synthesis as a whole must be considered, including the metrics shown on Table 2.

The two chemocatalytic routes rely on wasteful methylation steps which impact the greenness and efficiency of the synthesis to the point where it would not be commercialisable (Step 4 (S4) in Routes 1 and 2, Table 2). While AE and RME are better when methyllithium is used ( $92 \%$ and $63 \%$, respectively) compared to when a Grignard reagent is used ( $41 \%$ and $37 \%$ respectively), the former requires more stringent dry and air-free conditions.

This leaves Routes 3 and 4 as the favoured synthetic routes to TMO. Route 3 has the benefits of being an already established process for the production of DHL, albeit with petroleum-derived starting materials. It is also analogous to the early steps of the synthesis of NMP, suggesting that the economics of the process will not be an issue. Route 4 is uncommercialised to the best of our knowledge and requires extra steps. However, little or no purification is required at any stage of Route 4, benign conditions are required throughout, and yields are high. One difficulty with Route 4 is the synthesis of 2,5dimethylhexa-2,4-diene (DHN), Step 4, as the producer, Gevo, no longer advertise it on their product catalogue.

Overall, Route 3 is likely to be the best route to TMO due to it being an established process, requiring five steps from glucose, and having relatively good CHEM21 metrics (Table 2 and the supplementary CHEM21 spreadsheet) and BioLogic scores (Figure 1). In addition, the bio-based platform molecules used in the synthesis are acetone and methane; two of the most established bio-based molecules. Despite this assessment, it was only from Routes 1 and 2 that a sample of partially bio-based TMO could be prepared at this moment in time. Samples gathered from Route 1 were assessed via ASTM D6866-20 Method B and showed $64 \%$ bio-based carbon content in both the DHL and TMO subsequently produced.

\section{Conclusions}

Several routes to bio-based 2,2,5,5-tetramethyloxolane (TMO) have been proposed and assessed for greenness using the CHEM21 Metrics Toolkit and BioLogicTool. Two routes proceed chemocatalytically and two routes proceed predominately biochemically. TMO with a bio-based content of $64 \%$ was obtained by the methylation of bio-based methyl levulinate, surpassing the $25 \%$ required by the CEN/TC 411 bio-based content 
standard. However, despite high yields (90\%), atom economy, and process mass efficiency, stoichiometric amounts of magnesium salts are produced as a waste stream, meaning the coupling of acetone and acetylene is currently likely to be the greenest synthesis option, assuming bio-based acetone and acetylene are used. This study not only highlights the most logical pathway to bio-based TMO but also acts as an exemplar for the utility of the CHEM21 Metrics Toolkit and the BioLogicTool in comparing differing pathways to target bio-based compounds.

Supplementary Materials: The following are available online at https: / www.mdpi.com/article / 10.3390/suschem2030023/s1, Figure S1: 1H NMR spectrum of partially bio-based 2,2,5,5- tetramethyloxolane (TMO) produced by Route 1. CDCl3 solvent, Figure S2. 1H NMR spectrum of partially bio-based 2,5-dimethyl-2,5-hexandiol (DHL) produced by Route 1, d-MeOD solvent, Figure S3. 13C NMR spectrum of partially bio-based 2,5-dimethyl-2,5-hexandiol (DHL) produced by Route 1, dMeOD solvent, Figure S4. 1H NMR of 2,5-dimethyl-2,5-hexanediol as produced by Route 2. CDCl3 solvent, Figure S5. 13C NMR of 2,5-dimethyl-2,5-hexanediol as produced by Route 2, CDCl3 solvent, Figure S6. 1H NMR spectrum of 2,5-dimethylhexane-2,4-diene, d-MeOD solvent, Figure S7. 1H NMR spectrum of the polyperoxide of 2,5-dimethylhexane-2,4-diene (DHN polyperoxide). CDCl3 solvent, Figure S8. ASTM D6866-20 Method B for bio-based carbon testing results for 2,5-dimethyl2,4-hexanediol, Figure S9. ASTM D6866-20 Method B for bio-based carbon testing results for TMO. A supplementary spreadsheet titled "CHEM21 Metrics-All Routes" is the CHEM21 Metric Toolkit populated with available data to access the metrics for each proposed route with a separate tab for each step of each route, this was used to compiled the data given in Table 2 of the main manuscript. A supplementary spreadsheet titled "BioLogicTool-All Routes" is the BioLogicTool spreadsheet available from the original 2019 article by Lie et al. [9] and has been populated with data for each route, this was used to compile Figure 1 of the main manuscript.

Author Contributions: Conceptualization, F.P.B. and T.J.F.; methodology, F.P.B. and T.J.F.; formal analysis, F.P.B. and T.J.F.; investigation, F.P.B.; resources, F.P.B., C.A. and E.d.J.; data curation, F.P.B.; writing-original draft preparation, F.P.B. and T.J.F.; writing-review and editing, all authors; visualization, F.P.B. and T.J.F.; supervision, T.J.F. and J.H.C.; project administration, T.J.F., E.d.J. and J.H.C.; funding acquisition, T.J.F., E.d.J. and J.H.C. All authors have read and agreed to the published version of the manuscript.

Funding: This project has received funding from the Bio-Based Industries Joint Undertaking under the European Union's Horizon2020 research and innovation programme under agreement No 745450. The publication reflects only the authors' views and the JU is not responsible for any use that may be made of the information it contains.

Institutional Review Board Statement: Not applicable.

Informed Consent Statement: Not applicable.

Data Availability Statement: All relevant supporting data can be found within the manuscript or the accompanying supplementary files, noting that separate files are available for the BioLogicTool plots and CHEM21 Metrics Toolkit.

Conflicts of Interest: The authors declare no conflict of interest.

\section{References}

1. Byrne, F.; Forier, B.; Bossaert, G.; Hoebers, C.; Farmer, T.J.; Clark, J.H.; Hunt, A.J. 2,2,5,5-Tetramethyltetrahydrofuran (TMTHF): A Non-Polar, Non-Peroxide Forming Ether Replacement for Hazardous Hydrocarbon Solvents. Green Chem. 2017, 19, 3671-3678. [CrossRef]

2. Iemhoff, A.; Sherwood, J.; McElroy, C.R.; Hunt, A.J. Towards Sustainable Kinetic Resolution, a Combination of Bio-Catalysis, Flow Chemistry and Bio-Based Solvents. Green Chem. 2018, 20, 136-140. [CrossRef]

3. Pellis, A.; Byrne, F.P.; Sherwood, J.; Vastano, M.; Comerford, J.W.; Farmer, T.J. Safer Bio-Based Solvents to Replace Toluene and Tetrahydrofuran for the Biocatalyzed Synthesis of Polyesters. Green Chem. 2019. [CrossRef]

4. Byrne, F.P.; Hodds, W.M.; Shimizu, S.; Farmer, T.J.; Hunt, A.J. A Comparison of the Solvation Power of the Green Solvent 2,2,5,5-Tetramethyloxolane versus Toluene via Partition Coefficients. J. Clean. Prod. 2019, 240, 118175. [CrossRef]

5. Churchill, B.; Acree, W.E.; Abraham, M.H. Abraham Model Correlation for Direct Water-to-2,2,5,5-Tetramethyloxolane Solute Transfer Partitioning Process Revisited. Phys. Chem. Liq. 2019, 58, 833-838. [CrossRef] 
6. Maas-Brunner, M.; Henkelmann, J.; Kaibel, G.; Kindler, A.; Knoll, C.; Rust, H.; Tragut, C.; Stroezel, M.; Rheude, U.; Lorenz, R.E.; et al. Method for Preparing Acetylene Alcohols and Their Secondary Products. U.S. Patent No. 6,956,141, 18 October 2005.

7. ReSolve Project. Available online: http:/ / resolve-bbi.eu/ (accessed on 13 October 2020).

8. McElroy, C.R.; Constantinou, A.; Jones, L.C.; Summerton, L.; Clark, J.H. Towards a Holistic Approach to Metrics for the 21st Century Pharmaceutical Industry. Green Chem. 2015, 17, 3111-3121. [CrossRef]

9. Lie, Y.; Ortiz, P.; Vendamme, R.; Vanbroekhoven, K.; Farmer, T.J. BioLogicTool: A Simple Visual Tool for Assisting in the Logical Selection of Pathways from Biomass to Products. Ind. Eng. Chem. Res. 2019, 58, 15945-15957. [CrossRef]

10. van der Waal, J.C.; de Jong, E. Avantium Chemicals: The High Potential for the Levulinic Product Tree. In Industrial Biorenewables; John Wiley \& Sons, Ltd.: Hoboken, NJ, USA, 2016; pp. 97-120. ISBN 978-1-118-84379-6.

11. Hassan, S.S.; Williams, G.A.; Jaiswal, A.K. Moving towards the Second Generation of Lignocellulosic Biorefineries in the EU: Drivers, Challenges, and Opportunities. Renew. Sustain. Energy Rev. 2019, 101, 590-599. [CrossRef]

12. van Putten, R.-J.; van der Waal, J.C.; de Jong, E.; Rasrendra, C.B.; Heeres, H.J.; de Vries, J.G. Hydroxymethylfurfural, A Versatile Platform Chemical Made from Renewable Resources. Chem. Rev. 2013, 113, 1499-1597. [CrossRef] [PubMed]

13. Galbe, M.; Zacchi, G. Pretreatment: The Key to Efficient Utilization of Lignocellulosic Materials. Biomass Bioenergy 2012, 46, 70-78. [CrossRef]

14. Kang, S.; Fu, J.; Zhang, G. From Lignocellulosic Biomass to Levulinic Acid: A Review on Acid-Catalyzed Hydrolysis. Renew. Sustain. Energy Rev. 2018, 94, 340-362. [CrossRef]

15. YXY. Avantium. Available online: https://www.avantium.com/technologies/yxy/ (accessed on 8 July 2021).

16. Monteith, E.R.; Mampuys, P.; Summerton, L.; Clark, J.H.; Maes, B.U.W.; McElroy, C.R. Why We Might Be Misusing Process Mass Intensity (PMI) and a Methodology to Apply It Effectively as a Discovery Level Metric. Green Chem. 2020, 22, 123-135. [CrossRef]

17. Filiciotto, L.; Balu, A.M.; Van der Waal, J.C.; Luque, R. Catalytic Insights into the Production of Biomass-Derived Side Products Methyl Levulinate, Furfural and Humins. Catal. Today 2018, 302, 2-15. [CrossRef]

18. Huang, Y.-B.; Yang, T.; Cai, B.; Chang, X.; Pan, H. Highly Efficient Metal Salt Catalyst for the Esterification of Biomass Derived Levulinic Acid under Microwave Irradiation. RSC Adv. 2015, 6, 2106-2111. [CrossRef]

19. Kläusli, T. AVA Biochem: Commercialising Renewable Platform Chemical 5-HMF. Green Process. Synth. 2014, 3, 235-236. [CrossRef]

20. Román-Leshkov, Y.; Barrett, C.J.; Liu, Z.Y.; Dumesic, J.A. Production of Dimethylfuran for Liquid Fuels from Biomass-Derived Carbohydrates. Nature 2007, 447, 982-985. [CrossRef]

21. Li, Y.; Lv, G.; Wang, Y.; Deng, T.; Wang, Y.; Hou, X.; Yang, Y. Synthesis of 2,5-Hexanedione from Biomass Resources Using a Highly Efficient Biphasic System. ChemistrySelect 2016, 1, 1252-1255. [CrossRef]

22. Zakoshansky, V.M. The Cumene Process for Phenol-Acetone Production. Pet. Chem. 2007, 47, 273-284. [CrossRef]

23. Howard, W.L. Acetone. In Kirk-Othmer Encyclopedia of Chemical Technology; John Wiley \& Sons: New York, NY, USA, 2011; pp. 1-15. ISBN 978-0-471-23896-6.

24. Crabtree, R.H. Aspects of Methane Chemistry. Chem. Rev. 1995, 95, 987-1007. [CrossRef]

25. Ni, Y.; Sun, Z. Recent Progress on Industrial Fermentative Production of Acetone-Butanol-Ethanol by Clostridium Acetobutylicum in China. Appl. Microbiol. Biotechnol. 2009, 83, 415-423. [CrossRef] [PubMed]

26. Sreekumar, S.; Baer, Z.C.; Pazhamalai, A.; Gunbas, G.; Grippo, A.; Blanch, H.W.; Clark, D.S.; Toste, F.D. Production of an Acetone-Butanol-Ethanol Mixture from Clostridium Acetobutylicum and Its Conversion to High-Value Biofuels. Nat. Protoc. 2015, 10, 528-537. [CrossRef] [PubMed]

27. Li, Y.; Chen, Y.; Wu, J. Enhancement of Methane Production in Anaerobic Digestion Process: A Review. Appl. Energy 2019, 240, 120-137. [CrossRef]

28. Wang, P.; Wang, H.; Qiu, Y.; Ren, L.; Jiang, B. Microbial Characteristics in Anaerobic Digestion Process of Food Waste for Methane Production-A Review. Bioresour. Technol. 2018, 248, 29-36. [CrossRef] [PubMed]

29. Khoe Dinh, D.; Hoon Lee, D.; Song, Y.-H.; Jo, S.; Kim, K.-T.; Iqbal, M.; Kang, H. Efficient Methane-to-Acetylene Conversion Using Low-Current Arcs. RSC Adv. 2019, 9, 32403-32413. [CrossRef]

30. Kang, H.; hoon Lee, D.; Kim, K.; Jo, S.; Pyun, S.; Song, Y.; Yu, S. Methane to Acetylene Conversion by Employing Cost-Effective Low-Temperature Arc. Fuel Process. Technol. 2016, 148, 209-216. [CrossRef]

31. Khoo, H.H.; Ee, W.L.; Isoni, V. Bio-Chemicals from Lignocellulose Feedstock: Sustainability, LCA and the Green Conundrum. Green Chem. 2016, 18, 1912-1922. [CrossRef]

32. Moncada, J.; Posada, J.A.; Ramírez, A. Comparative Early Stage Assessment of Multiproduct Biorefinery Systems: An Application to the Isobutanol Platform. Bioresour. Technol. 2017, 241, 44-53. [CrossRef]

33. Ardolino, F.; Parrillo, F.; Arena, U. Biowaste-to-Biomethane or Biowaste-to-Energy? An LCA Study on Anaerobic Digestion of Organic Waste. J. Clean. Prod. 2018, 174, 462-476. [CrossRef]

34. Bartholomé, E. The BASF-Process for Production of Acetylene by Partial Oxidation of Gaseous Hydrocarbons. Chem. Eng. Sci. 1954, 3, 94-104. [CrossRef]

35. Taylor, J.D.; Jenni, M.M.; Peters, M.W. Dehydration of Fermented Isobutanol for the Production of Renewable Chemicals and Fuels. Top. Catal. 2010, 53, 1224-1230. [CrossRef] 
36. Sadri, F.; Ramazani, A.; Massoudi, A.; Khoobi, M.; Azizkhani, V.; Tarasi, R.; Dolatyari, L.; Min, B.-K. Magnetic CoFe $2 \mathrm{O}_{4}$ Nanoparticles as an Efficient Catalyst for the Oxidation of Alcohols to Carbonyl Compounds in the Presence of Oxone as an Oxidant. Bull. Korean Chem. Soc. 2014, 35, 2029-2032. [CrossRef]

37. Lakouraj, M.M.; Aghajani, B.; Mokhtary, M. Polyvinylpyrrolidone-Supported Hydroperoxide for Selective Oxidation of Aldehydes to Carboxylic Acids and Sulfides to Sulfoxides. Phosphorus Sulfur Silicon Relat. Elem. 2010, 185, 2393-2401. [CrossRef]

38. Evans, T.I.; Karas, L.J.; Rameswaran, R. Isobutylene Oligomerization Using Isooctane Diluent. U.S. Patent No. 5,877,372, 2 March 1999.

39. Peters, M.W.; Taylor, J.D.; Jenni, M.; Manzer, L.E.; Henton, D.E. Integrated Process to Selectively Convert Renewable Isobutanol to P-Xylene. U.S. Patent Application No. 12/899,285, 14 April 2011.

40. Taylor, T.J.; Taylor, J.D.; Peters, M.W.; Henton, D.E. Variations on Prins-like Chemistry to Produce 2,5-Dimethylhexadiene from Isobutanol. U.S. Patent No. 8,742,187, 3 June 2014.

41. Griesbaum, K.; Oswald, A.A.; Naegele, W. Polymeric Peroxide of 2,5-Dimethyl-2,4-Hexadiene and a New Selective Reduction of Its Peroxide Linkage. J. Org. Chem. 1964, 29, 1887-1892. [CrossRef] 